among urology specialists, male healthcare providers, practitioners who ordered PSA tests infrequently, and those affiliated with specific hospitals on univariate analysis, with the last two factors remaining significant on multivariate analysis $(P<0.05)$. The increasing age of male healthcare providers was significantly associated with an increasing level of inappropriate screening $(P<0.001)$.

Original article Kerfoot BP et al. (2007) Practitioner-level determinants of inappropriate prostate-specific antigen screening. Arch Intern Med 167: 1367-1372

\section{Antimicrobial prophylaxis in children with recurrent urinary tract infections}

A recent study published in JAMA reports that prophylactic antimicrobials increase the risk of antimicrobial resistance without decreasing the risk of recurrent urinary tract infections (UTIs) in a pediatric primary care cohort. Very little evidence exists regarding the risk factors associated with recurrent UTIs and the influence of antimicrobial prophylaxis in this setting.

Conway and colleagues from the US assembled a cohort of 83 children aged $\leq 6$ years who suffered from recurrent UTIs, from 27 centers across three states. The researchers performed time-to-event analysis to establish risk factors for recurrent UTIs and to study the effect of antimicrobial prophylaxis. They also performed a nested case-control study to determine risk factors for the development of antimicrobial resistance.

The results showed that white race, an age of 3-5 years, and grade 4-5 vesicoureteral reflux were risk factors for recurrent UTIs, whereas sex and grade 1-3 vesicoureteral reflux were not. There was no significant association between antimicrobial prophylaxis and a lower risk of recurrent UTI; however, antimicrobial prophylaxis did increase the risk of resistant infections in children with recurrent UTIs.

While this is the first study to examine the risks and benefits of antimicrobial prophylaxis for children with recurrent UTIs in a primary care setting, the authors highlight the limitations inherent in observational studies and stress the need for further research into this issue, particularly randomized controlled trials conducted in the community setting.

Original article Conway PH et al. (2007) Recurrent urinary tract infections in children: risk factors and association with prophylactic antimicrobials. JAMA 298: 179-186

\section{Oral antibiotics alone can be used to treat pediatric acute pyelonephritis}

Current guidelines recommend that children with acute pyelonephritis are initially treated with a parenteral third-generation cephalosporin such as ceftriaxone, and then oral antibiotics. A recent noninferiority trial has found, however, that oral antibiotics alone are just as effective as the recommended regime, a finding that will potentially lower costs and reduce the discomfort of children with pyelonephritis.

The randomized controlled trial recruited 502 children (aged 1 month to 7 years) with a first episode of acute pyelonephritis from 28 pediatric units across northeast Italy. Children were randomized to either parenteral ceftriaxone $50 \mathrm{mg} / \mathrm{kg} /$ day for 3 days followed by oral co-amoxiclav $50 \mathrm{mg} / \mathrm{kg} /$ day for 7 days ( $n=258$ ), or to co-amoxiclav $50 \mathrm{mg} / \mathrm{kg} /$ day for 10 days $(n=244)$.

The rates of scintigraphy-detected renal scarring at 12 months (examined in 400 children) were similar in patients who had received parenteral ceftriaxone plus oral co-amoxiclav and in those who had received oral co-amoxiclav only ( $17.7 \%$ and $13.7 \%$, respectively). Secondary outcomes, which assessed the efficacy of antibiotic treatment in the short term (i.e. reduction in inflammatory indices, percentage of patients with sterile urine $72 \mathrm{~h}$ after treatment initiation, and time to fever subsidence) were also similar in the two groups. After 3 days of treatment, urine cultures collected from 388 of 390 patients were negative; two cases (one in each treatment group) were positive for Pseudomonas aeruginosa. Minor adverse effects (mostly diarrhea or vomiting) occurred in 15 children who received initial treatment with co-amoxiclav; three patients experienced minor adverse effects with ceftriaxone.

Original article Montini G et al. (2007) Antibiotic treatment for pyelonephritis in children: multicentre randomised controlled non-inferiority trial. BMJ [doi:10.1136/ bmj.39244.692442.55] 